\title{
Reversibility of experimental rabbit liver cirrhosis by portal collagenase administration
}

\author{
Bo Jin ${ }^{1,2}$, Harvey J Alter ${ }^{2}$, Zhi-Cheng Zhang ${ }^{3}$, J Wai-Kuo Shih ${ }^{2}$, Juan M Esteban ${ }^{2, *}$, \\ Tao Sun ${ }^{3}$, Yun-Sheng Yang ${ }^{1}$, Qi Qiu ${ }^{2}$, Xiao-Lin Liu ${ }^{3}$, Lin Yao ${ }^{3}$, Hai-Dong Wang ${ }^{3}$ and \\ Liu-Fang Cheng ${ }^{1}$
}

${ }^{1}$ Department of Digestive Diseases, Military Medical Graduate School, Beijing, China; ${ }^{2}$ Infectious Disease
Section, Department of Transfusion Medicine, Warren G Magnuson Clinical Center, National Institutes of
Health, Bethesda, MD, USA and ${ }^{3}$ Naval General Hospital, Beijing, China

The regression of cirrhosis is associated with increased intrahepatic collagenolytic enzyme activity. We investigated whether collagenase supplementation via portal vein infusion can retard cirrhosis development and/or reverse cirrhosis. In all, 35 rabbits were initially assigned to study. However, because of high surgical mortality and infection, only 15 animals completed study. Four normal controls (group I) received olive oil subcutaneously (SC) for 12 weeks followed by normal saline portal perfusion for 12 weeks. Four (group II) received $\mathrm{CCl}_{4} \mathrm{SC}$ for 6 weeks followed by portal vein collagenase, $6 \mathrm{mg}$ twice weekly, plus SC $\mathrm{CCl}_{4}$ for 6 additional weeks and then killed. Four rabbits (group III) received $\mathrm{CCl}_{4} \mathrm{SC}$ for 12 weeks and then $6 \mathrm{mg}$ of collagenase portally for 12 weeks, while three control rabbits (group IV) received $\mathrm{CCl}_{4}$ for 12 weeks followed by saline for 12 weeks. After 12 weeks of $\mathrm{CCl}_{4}$, liver hydroxyproline content of collagenase-treated group II $(361.1 \pm 106.6 \mu \mathrm{g} / \mathrm{g})$ was significantly reduced compared with group III + IV that had not yet received collagenase $(589.0 \pm 162.9 \mu \mathrm{g} / \mathrm{g} ; P<0.05)$. In the main comparison, hydroxyproline content of collagenase-treated group III $(177.5 \pm 35.6 \mu \mathrm{g} / \mathrm{g})$ was significantly decreased compared with saline-treated controls $(446.3 \pm 150.1 \mu \mathrm{g} / \mathrm{g}$; $\boldsymbol{P}<\mathbf{0 . 0 1 )}$. Further, liver histology showed complete regression of cirrhosis in the collagenase-treated animals. No toxicity of liver, kidney, lung, brain or heart was observed histologically. Anaphylaxis occurred in 2/35 original animals (one fatal). In conclusion, this study provides 'proof of principle' that collagenase portal administration can retard cirrhosis development and speed regression of established cirrhosis in the rabbit $\mathrm{CCl}_{4}$ model. Potential application to humans is premature, but feasible, if these findings are confirmed in additional animal studies.

Laboratory Investigation (2005) 85, 992-1002. doi:10.1038/labinvest.3700304; published online 20 June 2005

Keywords: liver cirrhosis; experimental; therapeutics; collagenase; portal vein; rabbit; carbon tetrachloride

Liver cirrhosis is a major health problem worldwide. It represents the end stage and life-threating consequence of most inflammatory chronic liver diseases. Although patients with liver cirrhosis may remain asymptomatic for years, progressive deterioration is inevitable, and once complications develop, the mortality exceeds $50 \%$ in 5 years. ${ }^{1}$ The remarkable pathological characteristic of liver cirrhosis is the excessive extracellular matrix (ECM) accumulation in liver parenchyma, especially

Correspondence: Dr B Jin, MD and Dr HJ Alter, MD, Infectious Diseases Section, Department of Transfusion Medicine, Warren G Magnuson Clinical Center, Bldg. 10, Rm. 1C711, National Institutes of Health, Bethesda, MD 20892-1184, USA.

E-mails: bjin@cc.nih.gov, halter@cc.nih.gov

${ }^{*}$ Current address: Department Medicina Interna, Liver Unit, Hospital General Vall D'Hebron, Barcelona, Spain.

Received 22 February 2005; revised 10 May 2005; accepted 13 May 2005; published online 20 June 2005 around the sinusoids, portal and central veins, with nodule formation and impaired liver function. The only available therapy for liver cirrhosis is liver transplantation. However, limited liver donor availability renders this therapy unsuitable for a large number of potential recipients.

There is accumulating evidence from animal models, as well as patients, that liver fibrosis is reversible ${ }^{2-6}$ However, the reversibility of cirrhosis is controversial. Although there are some reports of cirrhosis regression, ${ }^{3,7-9}$ the reversal was generally considered incomplete. ${ }^{10}$ Intensive efforts to develop therapies to induce regression of liver fibrosis or cirrhosis have thus far been unsuccessful. Therapeutic models for diminishing liver fibrosis include: (a) etiological therapy to cure the primary disease or reduce the inflammation that precedes scarring; (b) reducing extracellular matrix production in liver parenchyma by decreasing the number or the 
function of activated hepatic stellate cells (HSC) either by downregulation of the HSC activation or stimulation of HSC apoptosis; (c) degradation of accumulated scar matrix in liver parenchyma. Recently, it was reported that cirrhosis in both the rat carbon tetrachloride $\left(\mathrm{CCl}_{4}\right)$ cirrhosis model and the biliary duct ligation cirrhotic model can be reversed by administration of adenoviral vectors encoding the matrix metalloproteinase (MMP) sequence. ${ }^{11-13}$ However, in addition to the concerns about their long-term biological safety, active MMP enzyme expression cannot be handled efficiently in vivo.

Bacterial collagenase portal perfusion has been used ex vivo for isolation of liver cells by degrading collagen tissue and dispersing cells. The current study examines whether limited dosage of collagenase in vivo could properly degrade excessive collagen tissue in cirrhotic liver and reverse cirrhosis without dispersing or disrupting liver cells. Bacterial collagenase was delivered directly to the $\mathrm{CCl}_{4}$-induced cirrhotic liver of rabbits via a permanent portal vein catheterization. It was found that the portal administration of collagenase can both delay cirrhosis development and reverse wellestablished liver cirrhosis in rabbits.

\section{Materials and methods}

\section{Equipments, Anesthetics, and Reagents}

Ketamine hydrochloride injection was a product of Shanghai First Pharmaceutical, China. Animal anesthetic compound injection Su-Mian-Xin composed of dihydroetorphine hydrochloride, dimethylaniline thiazole, ethylenediaminetetraacetic acid, and haloperidol, was from Veterinary Institute of Military Supplies University, China. The anesthetic compound used in this experiment was a mixture composed of one vial of ketamine hydrochloride $(0.1 \mathrm{~g} / 2 \mathrm{ml})$ and one vial of Su-MianXin animal anesthetic compound $(1 \mathrm{ml})$. Intramuscular injection of $0.6-0.8 \mathrm{ml}$ of this mixture can cause complete anesthesia for an adult rabbit, $1.5-2.0 \mathrm{ml}$ of this mixture can result to death of a rabbit.

The disposable sterile epidural anesthetic catheter was purchased from Shaanxi Zhijun Kemao Medical Instruments Inc., China. The injection cap was from B Braun Melsungen AG, Germany.

Collagenase type IV from Sigma (USA), with activity of $388 \mathrm{U} / \mathrm{mg}$, is composed of at least seven distinct collagenases with minor neutral protease activity. ${ }^{14-16}$ Heparin sodium injection was a product of Beijing Pharmaceutical, China. Chloramine $\mathrm{T}$ and dimethylaminobenzaldehyde were purchased from Merck (Germany). Olive oil, $\mathrm{CCl}_{4}$, L-hydroxyproline, potassium hydroxide, potassium chloride, phenolphthalein, sodium thiosulfate, chloric acid, borate acid, alanine, and toluene were from Beijing Chemical Reagents Company, China.

\section{Animals}

In all, 35 male New Zealand white rabbits weighing $1.7-2.2 \mathrm{~kg}$ were housed in approved animal care facilities with free access to water and standard rabbit chow, approved by the animal care committee of the Chinese Military Medical Graduate School. The rabbits were randomly divided into four groups (Figure 1). Group I: Five rabbits were treated as normal controls receiving olive oil by subcutaneous injection twice weekly at a dosage of $0.3 \mathrm{ml} / \mathrm{kg}$ body weight for the first 2 weeks and $0.2 \mathrm{ml} / \mathrm{kg}$ thereafter until the end of 12 weeks. Portal perfusion with normal saline was started at week 13 just after the cessation of olive oil injection, and given twice weekly for another 12 weeks. Group II: 11 rabbits were administered $50 \% \mathrm{CCl}_{4}$ in olive oil by subcutaneous injection twice weekly at a dosage of $0.3 \mathrm{ml} / \mathrm{kg}$ body weight for the first 2 weeks and $0.2 \mathrm{ml} / \mathrm{kg}$ body weight for another 10 weeks. Portal collagenase perfusion was started at the 7 th week, and given twice weekly until cessation of $\mathrm{CCl}_{4}$ administration at week 13 at which time the animals were killed by high-dose anesthesia. Group III: 11 rabbits were administered $50 \% \mathrm{CCl}_{4}$ in olive oil

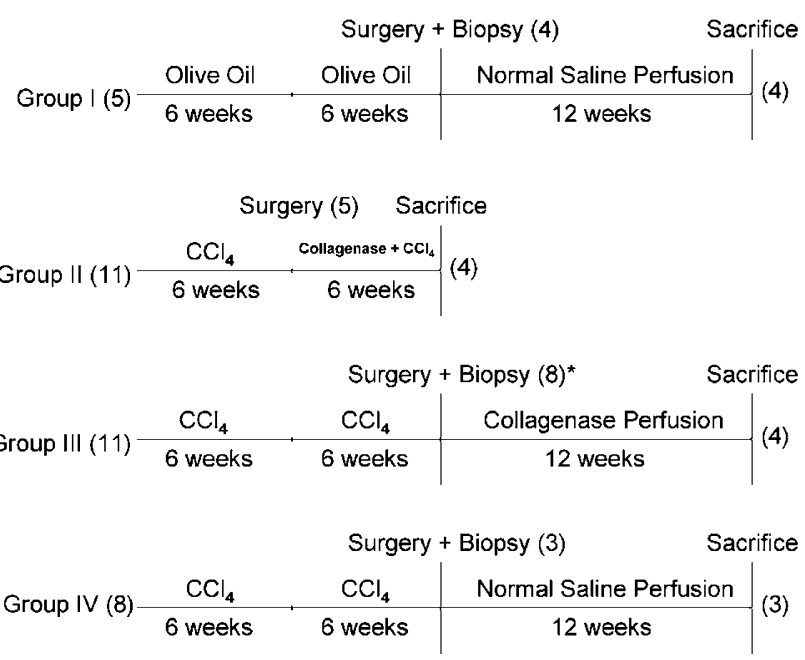

Figure 1 Group I: Normal control: after subcutaneous injection of olive oil twice weekly for 12 weeks, animals underwent portal catheterization and liver biopsy. Liver was perfused with normal saline twice weekly for another 12 weeks. Group II: Preventive treatment: animals were intoxicated with $50 \% \mathrm{CCl}_{4}$ twice weekly for 6 weeks, and then underwent portal catheterization and simultaneous portal collagenase perfusion and twice weekly SC administration of $50 \% \mathrm{CCl}_{4}$ for another 6 weeks. Group III: Cirrhosis regression: after subcutaneous injection of $50 \% \mathrm{CCl}_{4}$ for 12 weeks, animals were subjected to portal catheterization and liver biopsy. Liver was perfused with collagenase twice weekly for another 12 weeks without $\mathrm{CCl}_{4}$ toxicity. Group IV: Treatment control: after subcutaneous injection of $50 \% \mathrm{CCl}_{4}$ for 12 weeks, rabbits were subjected to portal catheterization and liver biopsy. Liver was perfused with normal saline twice weekly for another 12 weeks without $\mathrm{CCl}_{4}$ toxicity. The number in parentheses indicates the survived rabbits at the indicated time point. *: One rabbit was excluded from the experiment because of allergy to collagenase. 
twice weekly similar to group II. Portal collagenase perfusion was started at week 13 just after the cessation of $\mathrm{CCl}_{4}$ exposure, and given twice weekly for another 12 weeks without $\mathrm{CCl}_{4}$. Group IV: Eight rabbits were injected with $50 \% \mathrm{CCl}_{4}$ in olive oil twice weekly as in group II. Normal saline portal perfusion was started at week 13 and given twice weekly for another 12 weeks without $\mathrm{CCl}_{4}$. The rabbits in groups I, III, and IV were killed by highdose anesthesia at week 25 after 12 weeks of $\mathrm{CCl}_{4}$ exposure and another 12 weeks of portal perfusion.

Blood samples were taken from the inferior vena cava just prior to killing for detection of serum levels of alanine aminotransferase (ALT), aspartate aminotransferase (AST), bilirubin, albumin, urea, and creatinine using Beckman Coulter Synchron LX ${ }^{\mathbb{R}} 20$ Clinical System (Beckman Coulter Inc., USA). After sacrifice, the liver, kidney, and lung were sampled and fixed with $4 \%$ buffered formaldehyde, embedded in paraffin, sectioned in $5 \mu \mathrm{m}$, slides stained with $\mathrm{H} \& \mathrm{E}$ and Masson trichrome, and examined independently by two experienced pathologists. Another liver sample was taken and snap frozen in liquid nitrogen and stored in capped tubes at $-70^{\circ} \mathrm{C}$ for later hydroxyproline assay.

\section{Surgical Procedure}

All rabbits were subjected to surgery for permanent portal catheterization 2-9 days prior to portal perfusion. Surgery was performed through an upper abdominal mid-line incision of $5 \mathrm{~cm}$ length. A 0.1$0.2 \mathrm{~cm}^{3}$ liver sample was taken during the procedure, and divided into two parts. One was fixed with $4 \%$ buffered formaldehyde for pathological examination, the other was snap frozen in liquid nitrogen and stored in capped tubes at $-70^{\circ} \mathrm{C}$ for hydroxyproline assay. After liver biopsy, the jejunal mesenteric vein was uncovered and an incision was made after isolation of a terminal branch of the vein. The distal end of the vein was ligated, and a disposable sterile epidural anesthetic catheter with a sealed tip and three distal side-holes was filled with normal saline containing heparin $125 \mathrm{U} / \mathrm{ml}$ and then connected to a syringe containing 3-4 ml heparin saline. The catheter was threaded through the vessel incision into the proximal vein towards the liver, and when sufficient length of catheter $(3-4 \mathrm{~cm})$ was in the vein, it was fixed to the vessel by ligation. After ensuring the catheter was in the right position by injection of heparin saline without resistance and unimpeded aspiration of blood, the syringe was taken off and the catheter was cut into a suitable length $(12-15 \mathrm{~cm})$. The open end of the catheter was firmly attached to a connector with an injection cap immediately after cut down. The abdominal cavity was closed by suture, and the open end of the catheter with the injection cap filled with heparin saline was embedded subcutaneously on the right side of the abdominal wall. Thus, the portal perfusion could be performed by puncturing the injection cap through the skin.

\section{Collagenase Portal Perfusion}

The commonly used collagenase concentration for ex vivo liver cell isolation is $0.05 \%$. We estimated that a comparable in vivo dose to disperse cells in a rabbit liver would be $100-150 \mathrm{mg}$ in a volume of $200-300 \mathrm{ml}$. Since such a dosage is considered too high to be used therapeutically in vivo, for safety considerations, we selected $1 / 24$ of this ex vivo dosage or $6 \mathrm{mg}$ per infusion as a testable compromise between efficacy and tolerability. After 12 weeks of twice weekly administration the total amount infused, $144 \mathrm{mg}$, would be similar to the established ex vivo application of this methodology.

For determination of a safe dosage of collagenase, three rabbits were given collagenase portal perfusion at a dosage of 2,4 , or $6 \mathrm{mg} / \mathrm{rabbit}$ in $20 \mathrm{ml}$ normal saline, respectively, and killed $24 \mathrm{~h}$ after the enzyme administration. Blood samples were taken from the ear margin vein prior to and postcollagenase perfusion for routine blood, liver function, and renal function tests. After killing, the liver, myocardium, kidney, lung, and brain were sampled and fixed with $4 \%$ buffered formaldehyde for histological examination.

After estimating the tolerated dosage, $6 \mathrm{mg}$ collagenase type IV was dissolved in $20 \mathrm{ml}$ of sterile normal saline and portal perfusion was performed by puncturing the injection cap. The perfusion rate was set at about $2 \mathrm{ml} / \mathrm{min}$. The enzyme was administered twice weekly. After perfusion, $5 \mathrm{ml}$ of normal saline containing $125 \mathrm{U} / \mathrm{ml}$ heparin was injected into the catheter to prevent catheter obstruction.

\section{Hydroxyproline Assay}

This assay was conducted according to the method of Kivirikko et $a l^{17}$ with minor modifications. ${ }^{18}$

\section{Statistical Analysis}

Data are expressed as mean \pm s.d. One-way analysisof-variance was used to assess the differences in serological data and liver hydroxyproline content among experimental groups except the difference of liver hydroxyproline content between pre- and postcollagenase/saline perfusion that was assessed by unpaired $t$-test by Stata 7.0 software. $P$-values of $<0.05$ were considered statistically significant.

\section{Results}

\section{$\mathrm{CCl}_{4}$ Injection and Animal Survival}

During initial surgery and the first 12 weeks of $\mathrm{CCl}_{4}$ exposure, there was a high mortality rate unrelated 
to collagenase treatment. Of the 35 animals assigned to the study, five rabbits in group II died during the initial surgery for catheter placement and one animal died later during catheter replacement. Group II was the first group to undergo surgery in this study and mortality reflected, at least in part, operator inexperience. Surgical mortality decreased in each successive phase of the study with two deaths observed in group III, one death in group IV and no deaths in group I, the last group to be studied. Six additional animals died of medical causes, primarily infection, during the first 12 weeks they were on study. One rabbit in group I died of severe otic infection. In sum, early mortality resulted in the loss of 16 of the $35(46 \%)$ initially assigned animals. Thus, prior to collagenase treatment (Figure 1) there were four remaining animals in group I (controls), five animals in group II, eight in group III (one living animal excluded because of allergy to collagenase) and three in group IV. Further mortality occurred during collagenase treatment with two animals in group III dying from infection secondary to extensive subcutaneous necrosis of the abdominal wall caused by leakage of collagenase from a corroded catheter. One additional animal in group III died of laryngospasm and paralysis after the first collagenase perfusion and was considered a death due to anaphylactic shock. Another animal in group III also sustained anaphylactic shock but recovered after adrenalin administration; nonetheless, this animal had to be removed from study. No other anaphylactic reactions were observed even after repeated intravenous collagenase injections. In the final analysis, 15 of the $35(43 \%)$ survived until the end of study at which time they were killed for morphologic analysis.

\section{Effect of Portal Collagenase Perfusion on Blood Cells and Other Organs}

Portal collagenase administration has the potential to injure liver cells, veins, blood cells, and even other organs such as kidney, heart, brain, and lung or to induce inflammation in the related tissue. For evaluating the safety of portal collagenase administration, we investigated liver and kidney function tests in rabbits $24 \mathrm{~h}$ after different dosages of collagenase perfusion. The results of blood tests suggested that collagenase portal perfusion had no significant effects on blood cells, liver function, and renal function (Table 1), as evidenced by stable pre- and postperfusion levels of white blood cell, erythrocyte and platelet counts, hemoglobin level, ALT, AST, bilirubin, albumin, blood urea nitrogen, and creatinine. Further, histology of brain, heart, liver, kidney, and lung from rabbits receiving 2, 4, and $6 \mathrm{mg}$ collagenase revealed no abnormal findings (data not shown).

After 6-12 weeks of collagenase portal perfusion, there were still no significant differences in serum levels of ALT, AST, bilirubin, total serum protein, urea, and creatinine among the experimental groups and normal controls (Table 2), suggesting that collagenase portal perfusion does not cause damage to the liver or kidney at the dosage used $(6 \mathrm{mg} /$ rabbit).

\section{Effects of Portal Collagenase Perfusion on Cirrhotic Liver Collagen Content}

Over the first 12 weeks of study, groups III and IV were identically treated with $\mathrm{CCl}_{4}$ without collagenase (Figure 1), whereas group II received 6 weeks of $\mathrm{CCl}_{4}$ followed by 6 weeks of $\mathrm{CCl}_{4}$ plus collagenase (Figure 1). Groups III and IV were compared with group II to determine if the 6 weeks of collagenase in group II lowered hydroxyproline content or impaired liver histology compared to animals that did not receive collagenase during that 6 -week period. Animals in group II were killed after 12 weeks whereas animals in groups III and IV received $\mathrm{CCl}_{4}$ only in these 12 weeks and portal perfusion for an additional 12 weeks. After 12 weeks, the liver hydroxyproline content of groups III and IV were

Table 1 Blood tests for safety evaluation

\begin{tabular}{|c|c|c|c|c|c|c|}
\hline \multirow[t]{2}{*}{ Test } & \multicolumn{2}{|c|}{$\begin{array}{l}\text { Rabbit with } 2 \text { mg collagenase } \\
\text { portal perfusion }(\mathrm{n}=1)\end{array}$} & \multicolumn{2}{|c|}{$\begin{array}{l}\text { Rabbit with } 4 \mathrm{mg} \text { collagenase } \\
\text { portal perfusion }(\mathrm{n}=1)\end{array}$} & \multicolumn{2}{|c|}{$\begin{array}{l}\text { Rabbit with } 6 \text { mg collagenase } \\
\text { portal perfusion }(\mathrm{n}=1)\end{array}$} \\
\hline & Preperfusion & Postperfusion & Preperfusion & Postperfusion & Preperfusion & Postperfusion \\
\hline White blood cells $\left(10^{9} / 1\right)$ & 9.5 & 7.6 & 9.2 & 5.3 & 4.4 & 7.1 \\
\hline Erythrocytes $\left(10^{12} / 1\right)$ & 4.17 & 4.02 & 6.18 & 5.68 & 5.15 & 5.33 \\
\hline Hemoglobin (g/dl) & 9.4 & 9.0 & 12.2 & 11.3 & 10.8 & 11.2 \\
\hline Platelets $\left(10^{9} / 1\right)$ & 46 & 200 & 292 & 419 & 106 & 101 \\
\hline ALT (IU/l) & 30 & 28 & 34 & 34 & 29 & 27 \\
\hline AST (IU/l) & 114 & 54 & 46 & 18 & 33 & 24 \\
\hline Bilirubin $(\mu \mathrm{mol} / \mathrm{l})$ & 0.7 & 2.6 & 3.3 & 1.3 & 1.6 & 1.5 \\
\hline Albumin (g/l) & 15 & 14 & 17 & 17 & 18 & 17 \\
\hline Urea (mmol/l) & 10.3 & 8.0 & 7.2 & 6.9 & 5.2 & 7.4 \\
\hline Creatine $(\mu \mathrm{mol} / \mathrm{l})$ & 139 & 91 & 73 & 72 & 77 & 94 \\
\hline
\end{tabular}


Table 2 Hepatic and renal function test (mean \pm s.d.)

\begin{tabular}{|c|c|c|c|c|c|c|}
\hline Group & $I(\mathrm{n}=4)$ & $I I(\mathrm{n}=4)$ & $I I I(\mathrm{n}=4)$ & $I V(\mathrm{n}=3)$ & F-value & $\mathrm{P}$-value \\
\hline ALT (IU/l) & $38.25 \pm 9.11$ & $35.25 \pm 6.18$ & $34.00 \pm 11.75$ & $40.67 \pm 16.01$ & 0.27 & 0.8473 \\
\hline AST (IU/l) & $45.25 \pm 6.29$ & $57.25 \pm 11.87$ & $54.50 \pm 15.84$ & $43.67 \pm 26.03$ & 0.69 & 0.5785 \\
\hline Bilirubin $(\mu \mathrm{mol} / \mathrm{l})$ & $0.62 \pm 0.53$ & $1.08 \pm 0.68$ & $0.43 \pm 0.22$ & $0.40 \pm 0.20$ & 1.65 & 0.2354 \\
\hline Total serum protein $(\mathrm{g} / \mathrm{l})$ & $67.00 \pm 10.36$ & $60.00 \pm 6.48$ & $65.50 \pm 9.98$ & $64.33 \pm 14.01$ & 0.35 & 0.7901 \\
\hline Urea $(\mathrm{mmol} / \mathrm{l})$ & $7.10 \pm 0.63$ & $8.33 \pm 1.37$ & $7.40 \pm 1.23$ & $9.77 \pm 5.36$ & 0.77 & 0.5343 \\
\hline Creatinine $(\mu \mathrm{mol} / \mathrm{l})$ & $136.75 \pm 15.20$ & $93.25 \pm 16.82$ & $131.50 \pm 36.32$ & $153.67 \pm 50.14$ & 2.49 & 0.1145 \\
\hline
\end{tabular}

Note: Blood samples of group II were taken at week 13 and groups I, III, and IV at week 25.

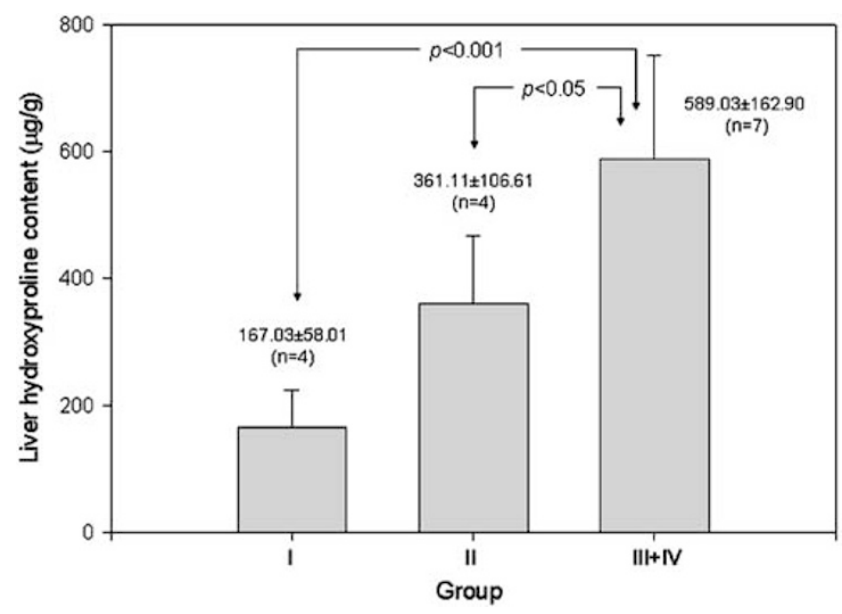

Figure 2 Rabbit liver hydroxyproline content after 12 weeks of $\mathrm{CCl}_{4}$ injection with or without 6 weeks of simultaneous portal collagenase perfusion (Mean \pm s.d.). Group I: Normal control; group II: rabbits received $\mathrm{CCl}_{4}$ injection for 6 weeks before the collagenase portal perfusion, and then received both the $\mathrm{CCl}_{4}$ injection and the collagenase perfusion simultaneously for another 6 weeks; Group III + IV: rabbits received $\mathrm{CCl}_{4}$ injection without collagenase for 12 weeks.

combined and compared to group II. Collagenasetreated group II rabbits showed significantly less hydroxyproline content $(361.11 \pm 106.61 \mu \mathrm{g} / \mathrm{g})$ in liver than untreated animals in groups III + IV $(589.03 \pm 162.90 \mu \mathrm{g} / \mathrm{g})(P<0.05)$ (Figure 2). However, this experiment does not exclude possible confounding effects of surgery and portal perfusion. While these procedures are unlikely to account for the observed differences, a control for group II that underwent surgery followed by saline perfusion rather than collagenase perfusion would be needed to exclude this possibility. Liver histology also revealed that the rabbits that received collagenase perfusion had less liver fibrosis (Figure $3 \mathrm{~b}$ ) than the nonperfused rabbits (Figure $3 \mathrm{c}$ and e). This result suggests that portal collagenase perfusion can decrease the liver collagen accumulation and retard the development of liver cirrhosis in rabbits intoxicated with $\mathrm{CCl}_{4}$.

After 12 weeks of either oil injection or $\mathrm{CCl}_{4}$ exposure, all the rabbits in groups I, III, and IV were subjected to surgery for liver biopsy and permanent portal catheterization. Liver histology showed that all $\mathrm{CCl}_{4}$ intoxicated rabbits developed liver cirrhosis after 12 weeks of exposure (Figure 3c and e). After a subsequent 12 weeks of collagenase portal administration to group III animals, the accumulated fibrous tissue diminished dramatically and the liver histology returned almost to normal (Figure 3d). Control rabbits receiving saline perfusion (group IV) exhibited a marked spontaneous regression in fibrosis during the 12 weeks after cessation of $\mathrm{CCl}_{4}$, but regression was incomplete and thin fibrous septa still persisted (Figure 3f). Assay of liver hydroxyproline content disclosed that the rabbits given collagenase (group III) had significantly decreased liver hydroxyproline content $(177.5 \pm 35.6 \mu \mathrm{g} / \mathrm{g})$ compared to $\mathrm{CCl}_{4}$-treated animals that did not receive collagenase (group IV) $(446.3 \pm 150.1 \mu \mathrm{g} / \mathrm{g})(P<0.01)$. Hydroxyproline content after collagenase treatment $(177.5 \pm 35.6 \mu \mathrm{g} / \mathrm{g})$ was similar to that of control animals (group I) that had not been exposed to $\mathrm{CCl}_{4}(153.2 \pm 22.3 \mu \mathrm{g} / \mathrm{g})$ whereas $\mathrm{CCl}_{4}$-treated animals that did not receive collagenase (group IV) had a significantly higher hydroxyproline contents $(446.3 \pm 150.1 \mu \mathrm{g} / \mathrm{g})$ than group I controls (Figure $4 \mathrm{a}, P<0.005)$. Comparing the liver hydroxyproline content of group III pre- and postcollagenase perfusion $(501.8 \pm 144.4$ Vs $177.5 \pm 35.6 \mu \mathrm{g} / \mathrm{g})$, showed a significant reduction $(P<0.05)$ whereas the control rabbits that received normal saline perfusion (group IV) had a reduction that did not reach significance $(705.4 \pm 113.2$ vs $446.3 \pm 150.1 \mu \mathrm{g} / \mathrm{g} ; P=0.124)$ (Figure $4 \mathrm{~b}$ ). It is notable that there was a difference between groups III and IV in liver hydroxyproline content even before portal perfusion $(705.4 \pm 113.2$ vs $501.8 \pm 144.4 \mu \mathrm{g} / \mathrm{g}, \quad P=0.101$ ), although these rabbits had received identical treatments up to that time. This difference was not significant and may reflect individual variation in response to $\mathrm{CCl}_{4}$ toxicity. These results suggest that collagenase portal administration can promote the regression of liver cirrhosis and reverse well-established liver cirrhosis to almost normal histology after the withdrawal of $\mathrm{CCl}_{4}$ toxicity in rabbits.

Due to the fact that liver inflammation precedes liver fibrogenesis in the $\mathrm{CCl}_{4}$ cirrhosis model, we compared the liver histology of animals that received $\mathrm{CCl}_{4}$ for 12 weeks combined with collagenase for the last 6 weeks (group II) with the liver histology 

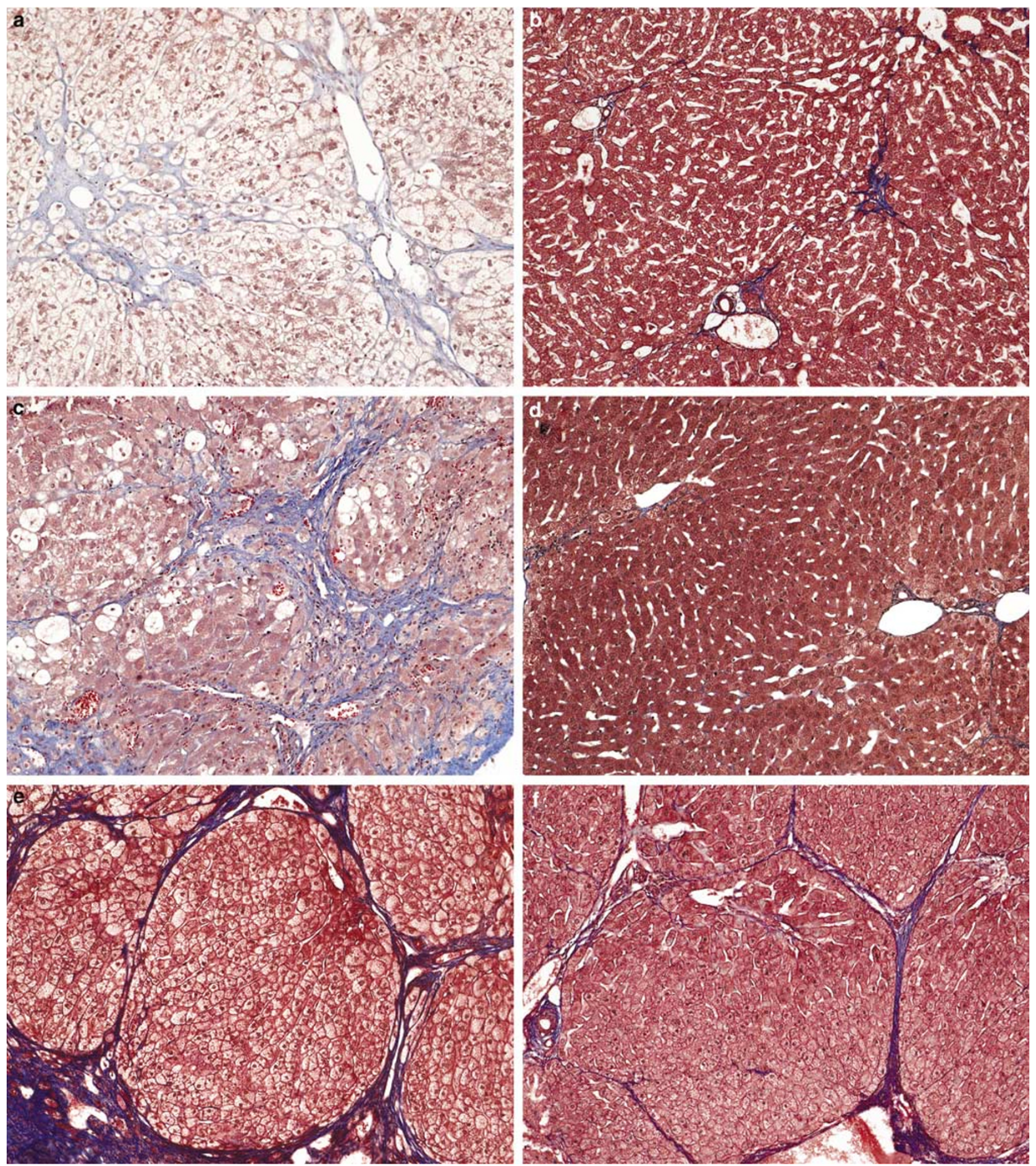

Figure 3 Liver histology pre- and postcollagenase portal perfusion. (a and b): Group II, rabbit liver histology after 6 weeks of CCl ${ }_{4}$ toxicity showed severe fibrosis (a). After another 6 weeks of collagenase portal perfusion together with $\mathrm{CCl}_{4}$ exposure, only slight fibrosis remained (b) compared with those without collagenase administration during weeks 6-12 (c and e). (c and d): Group III, rabbit liver histology after 12 weeks of $\mathrm{CCl}_{4}$ exposure showed established cirrhosis with liver cell degeneration (c). Following 12 weeks of collagenase portal administration, the fibrotic septa diminished dramatically and the liver tissue was restored to almost normal histology (d). (e and f): Group IV, rabbit liver histology after 12 weeks of $\mathrm{CCl}_{4}$ exposure showed established cirrhosis (e). After another 12 weeks of saline portal perfusion, the cirrhotic liver demonstrated spontaneous regression but the fibrotic septa still existed (f). (Stained with Masson trichrome, original magnification $10 \times 10$ ). 

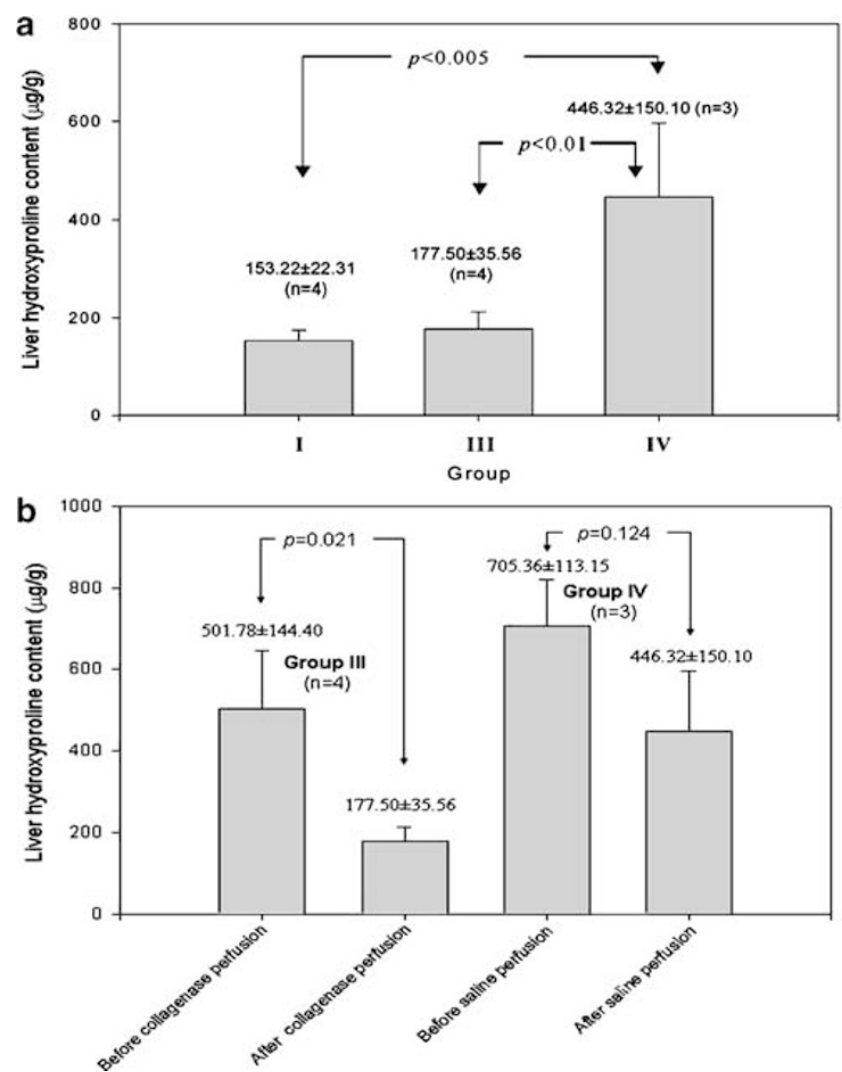

Figure 4 Liver hydroxyproline content according to collagenase treatment. (a) Portal collagenase administration (group III) significantly decreased the hydroxyproline content of cirrhotic rabbit liver (compared with the group IV controls $P<0.01$ ). After withdrawal of the toxin, the cirrhotic rabbit liver can regress and restore slowly, but after 12 weeks of saline administration (group IV), the liver hydroxyproline content was still significantly more than that of the normal (group I) $(P<0.005)$. whereas collagenase restored liver hydroxyproline to normal. (b) There was a significant reduction of liver hydroxyproline content in group III after collagenase portal perfusion $(P<0.05)$. The liver hydroxyproline content in group IV also displayed a decrease, though not statistically significant, after the withdrawal of $\mathrm{CCl}_{4}$, which represented spontaneous regression. Prior to collagenase or saline perfusion, the liver hydroxyproline content between groups III and IV were not significantly different $(P=0.124)$. Group I: Normal control; group III: rabbits that received $\mathrm{CCl}_{4}$ injection for 12 weeks, followed by collagenase portal perfusion for another 12 weeks; group IV: rabbits that received $\mathrm{CCl}_{4}$ injection for 12 weeks, followed by normal saline portal perfusion for another 12 weeks.

of animals that received $\mathrm{CCl}_{4}$ for 12 weeks without collagenase (groups III and IV). The exhibit of inflammation was similar in each of these groups, suggesting that collagenase did not inhibit inflammation (Figure 5) and thus did not exert its antifibrotic property through inhibition of hepatic inflammation. Instead, an early report suggested the haemorrhagic and inflammatory properties of collagenase. ${ }^{19}$ There were no pathological findings in liver, kidney, or lung of the rabbits administered collagenase portally (group III) (Figure 6b, d, and f) compared to that of the control rabbits (group IV) (Figure 6a, c, and e).

\section{Discussion}

Recent studies have provided considerable information about the regression of liver cirrhosis in animal models, as well as in patients, after the inciting agent or toxin was removed..$^{2-4,20,21}$ MMP in liver are the most important enzymes involved in liver cirrhosis regression. ${ }^{22}$ It has been well demonstrated that the MMP activity is diminished in cirrhotic liver whereas the inhibitory factor, tissue inhibitors of metalloproteinase, is increased. ${ }^{23,24}$ Failure to degrade the accumulated ECM due to excessive ECM deposition and relatively insufficient MMP activity is a potential mechanism for progression of fibrosis to cirrhosis. Regression of liver cirrhosis must be initiated by degradation of the excessive ECM accumulated in the liver, followed by the restoration of liver architecture. Therefore increased collagenolytic activity is critical to triggering cirrhosis regression.

Many attempts to increase the collagenolytic enzyme activity in cirrhotic liver have been undertaken. Some chemicals were reported to have the potential to increase MMP activity and thereby reverse liver fibrosis., ${ }^{6,25}$ Other studies delivered MMP DNA recombinant adenovirus vectors to fibrotic rat liver. ${ }^{11-13}$ The encoded enzyme was expressed in fibrotic rat liver and improved intrahepatic MMP activity, but hepatic gene delivery in humans would be difficult as would control of gene product expression. Additionally, there would be need to prevent the recombinant adenovirus from entering organs other than fibrotic liver and destroying the ECM framework of these normal organs. In this paper, we report the delivery of active collagenolytic enzyme directly to the cirrhotic liver through portal catheterization. In this method, enzyme activity in liver can be controlled by altering dosage administered, and the administration can be stopped at any time when the excessive ECM is properly degraded.

Bacterial collagenase from Clostridium histolyticum is a collagenolytic enzyme which can recognize and bind collagen-like sequences with repeated Gly-Pro-X, and degrade collagen into small peptides by hydrolyzing almost all of the Y-Gly bonds in collagen chains. ${ }^{26}$ Our measurement of liver hydroxyproline content as well as histological examination revealed that bacterial collagenase portal perfusion can efficiently delay liver cirrhosis development and hasten the regression of well-established liver cirrhosis in rabbits. However, it must be noted that $\mathrm{CCl}_{4}$-induced cirrhosis will spontaneously regress once the toxic injury is removed. Thus in this model one cannot measure cirrhosis regression in absolute terms, but can only measure the relative rate of regression in collagenase-treated or -untreated animals. However, if the fibrogenic stimuli continue, as in group II, collagenase perfusion can only delay cirrhosis development rather than prevent its occurrence. This would make 

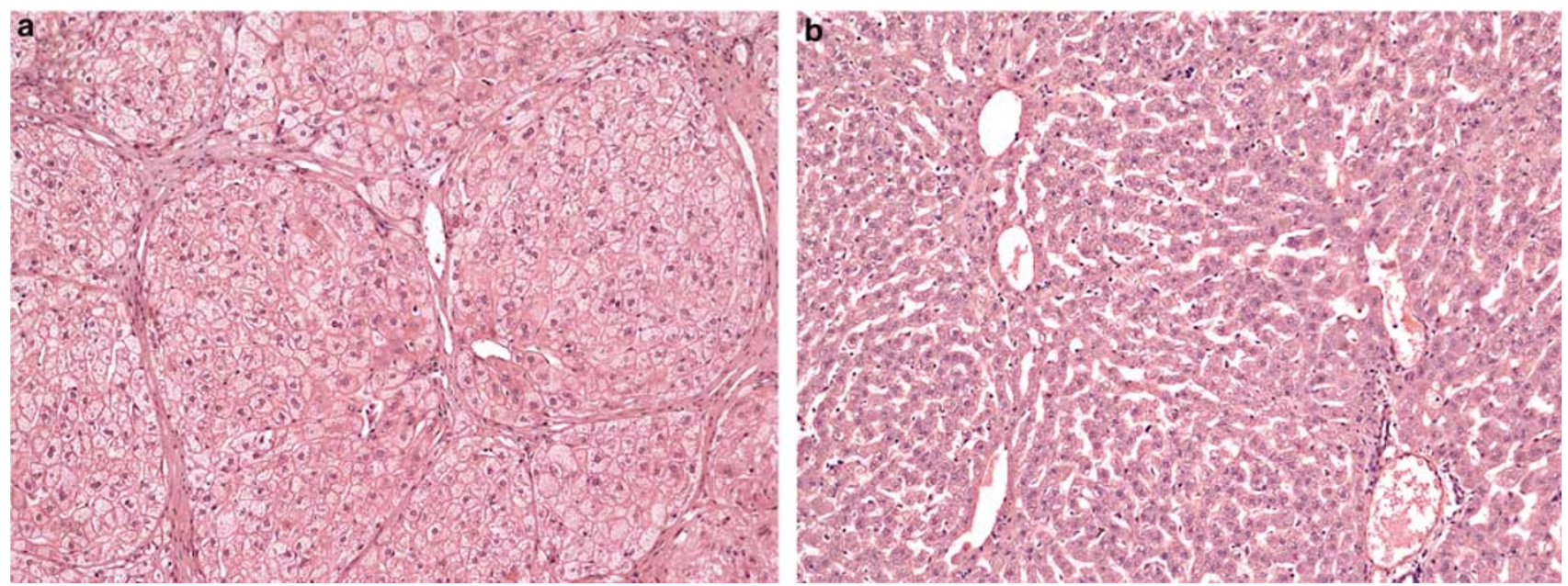

Figure 5 Histology of liver tissue after 12 weeks exposure of $\mathrm{CCl}_{4}$ with or without collagenase treatment. (a) Liver tissue of a rabbit from group IV that received 12 weeks of $\mathrm{CCl}_{4}$ without collagenase treatment. (b) Liver tissue of a rabbit from group II that received 12 weeks of $\mathrm{CCl}_{4}$ and 6 weeks of collagenase treatment. There was no discernable difference in the extent of hepatic inflammation in the liver between rabbits that received $\mathrm{CCl}_{4}$ with collagenase and animals that received $\mathrm{CCl}_{4}$ without collagenase. (Stained with $\mathrm{H} \& \mathrm{E}$, original magnification $10 \times 10$.)

collagenase treatment more applicable to cirrhosis patients where the injurious agent has been removed, such as in a recovered alcoholic or a successfully treated viral infection, rather than patients with ongoing injury from persistent infection or chronic autoimmune reaction. The possibility that prolonged collagenase administration could prevent or reverse cirrhosis development should be examined in future research. Considering that cirrhotic liver contains abundant collagen, it is assumed that most of the collagenase delivered portally will be bound to liver collagen, and thus not enter the systemic circulation where it could degrade collagen in organs other than the cirrhotic liver. This assumption was supported by our results that only the accumulated collagen in cirrhotic liver was dissolved; there was no evidence of collagen degradation in tissues other than liver.

Bacterial collagenase has been used clinically in the treatment of collagen disorders for many years. Early studies suggested that this collagenase can be used locally or systemically, ${ }^{27,28}$ and there was no evidence of systemic side effects, neural or vascular damage, or skin injury. ${ }^{27-30}$ An early study showed that in 44 patients receiving intrapenile injections of collagenase for treatment of Peyronie's disease, only one had detectable collagenase-specific IgE antibody in serum, and the author concluded that anaphylactic reaction to collagenase would be unlikely. ${ }^{31} \mathrm{In}$ this report, consistent with previous findings, no evidence was found for functional injury of liver or kidney, and no histological damage of heart, lung, liver, kidney, or brain was observed. There was also no evident blood cell injury. However, there were two rabbits that experienced anaphylactic shock and one of them died. It seems that collagenase can rarely induce a serious anaphylactic reaction, even on the first administration, and thus it will be safer to combine an antianaphylaxis regimen with bacterial collagenase portal administration in future studies. Alternatively, a recombinant mammalian interstitial collagenase, for example, MMP-1 or MMP-8, may be tolerated better than bacterial collagenase and used more safely in the treatment of liver cirrhosis. Our results also suggested that the dosage of $6 \mathrm{mg}$ of collagenase is harmful when it leaks out of the vessel since it can digest the subcutaneous connective tissue and cause extensive necrosis subcutaneously as two rabbits in group III died of this leakage in this experiment. Therefore, when this regimen is chosen for the treatment of liver cirrhosis, the quality of the implanted injection apparatus should be improved to ensure its integrity in vivo.

A previous report has suggested that collagenase portal infusion can increase the hepatocyte's competence for response to growth factors like hepatocyte growth factor or transforming growth factor- $\alpha .^{32}$ This increased mitogenic effect of growth factors induced by collagenase is of benefit for the regeneration and structural restoration of the cirrhotic liver after the excessive ECM has been removed by collagenase treatment. However, if this treatment approach is to be applied clinically in the future, liver malignancy must be ruled out because of the increased possibility of tumor proliferation and dissemination by collagenase perfusion.

In accordance with others' reports,,$^{4,23}$ our results revealed that, after withdrawal of the toxin, liver histology spontaneously improved within 3 months leaving only thin fibrous septa, although hepatic hydroxyproline content remained elevated. Although there is a slow spontaneous partial resolution of cirrhosis after cessation of $\mathrm{CCl}_{4}$, the portal 

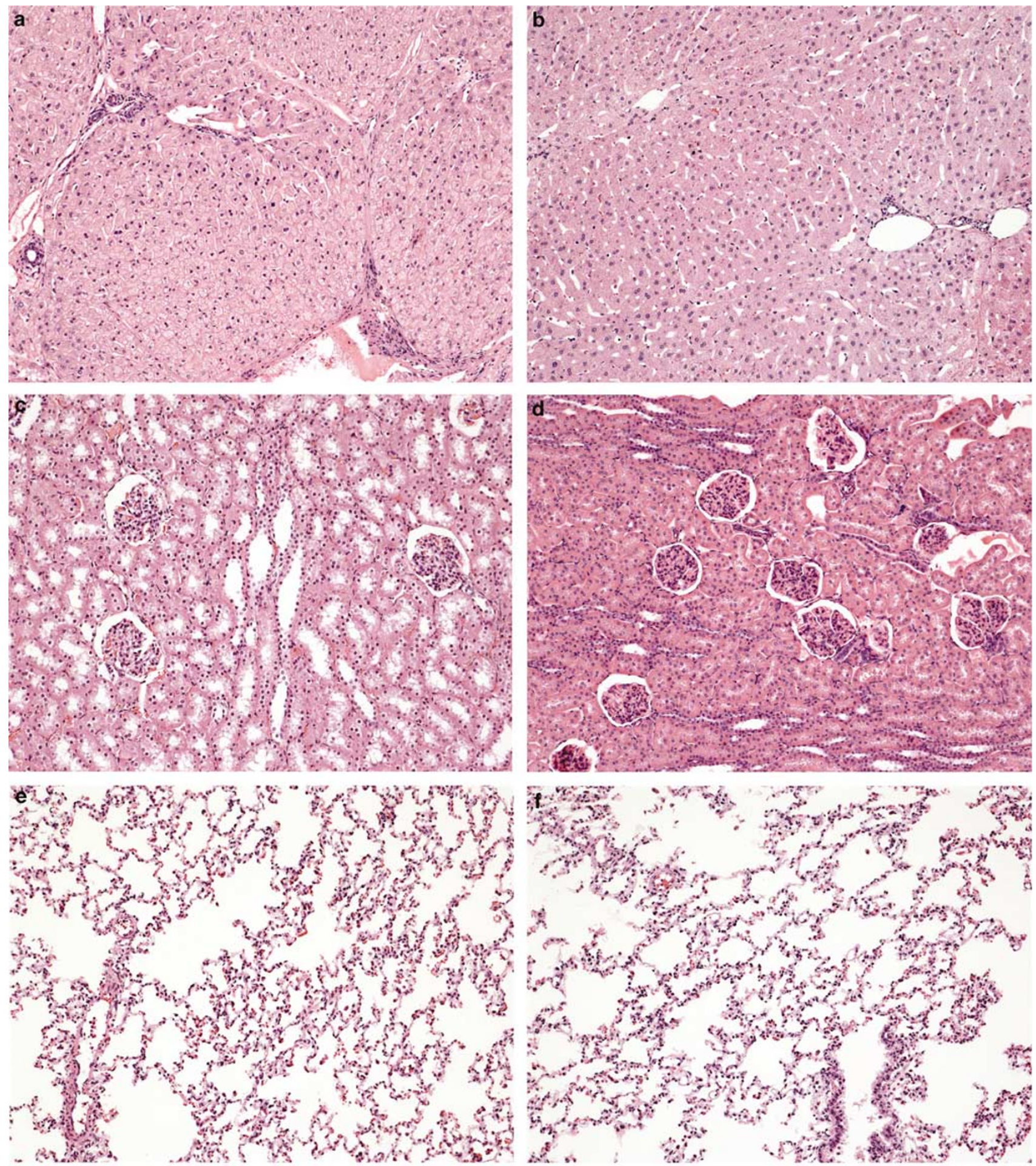

Figure 6 Histology of liver, kidney, and lung in rabbits treated with collagenase or saline. There were no abnormal findings in the liver (except the expected liver fibrosis), kidney, and lung of the rabbits (group III) portally administered collagenase (b, d, and f) for 12 weeks compared with that of the control rabbits (group IV) (a, c, and e) receiving saline portal perfusion. (Stained with H\&E, original magnification $10 \times 10$.)

administration of collagenase accelerated the resolution of the deposited ECM in cirrhotic liver, and after 3 months' administration, the liver histology was restored to almost normal architecture.
The $\mathrm{CCl}_{4}$ fibrotic rabbit model was utilized in the current study. $\mathrm{CCl}_{4}$ hepatic toxicity is primarily mediated by its metabolism in hepatocytes by monooxygenase through cytochrome P-450. The 
metabolites of $\mathrm{CCl}_{4}$, including the highly reactive radical ${ }^{\bullet} \mathrm{CCl}_{3}$ or ${ }^{\bullet} \mathrm{OOCCl}_{4}$, induce lipid peroxidation affecting plasma membrane, and result in pathological degeneration of liver cells. ${ }^{33}$ Previous reports suggested that the monooxygenase activity of the cytochrome P-450 system in hepatocytes declined after cells were isolated by collagenase perfusion..$^{34,35}$ However, the collagenase dosage used in hepatocyte isolation is much higher than the $6 \mathrm{mg}$ used in this study. It needs to be clarified whether the small dosage of collagenase used in our study could affect the monooxygenase activity of the cytochrome-P-450 system. Furthermore, results from groups III and IV demonstrated that collagenase promoted the regression of cirrhosis within 12 weeks following $\mathrm{CCl}_{4}$ exposure; this suggests that the antifibrotic effects of collagenase are unlikely to be mediated by inhibition of $\mathrm{CCl}_{4}$ bioactivation. For further clarification, other cirrhotic models than the $\mathrm{CCl}_{4}$ model should be selected to examine the antifibrotic effects of collagenase

Although animals that survived the full course of $\mathrm{CCl}_{4}$ exposure, portal vein surgery and collagenase treatment demonstrated significant reduction of liver hydroxyproline content and histologic fibrosis compared to control animals, this study was hampered by high surgical and infection-related mortality such that only $45 \%$ of animals completed study. The sequential decline in surgical mortality with each successive experiment exemplified a technical 'learning curve' and suggested that the problem could be substantially circumvented in future studies. Although the number of animals completing study was small, the results in each group were consistent and it was apparent that collagenase treatment had a significant effect on delaying the development of cirrhosis and promotion of cirrhosis regression. Another caveat of the study is that there was a spontaneous regression of cirrhosis after the toxin was removed such that collagenase effect in this model had to be balanced against spontaneous recovery. Collagenase-treated animals responded more rapidly and more completely than untreated controls, but both groups showed improvement after stopping $\mathrm{CCl}_{4}$ administration so that differences were quantitative rather than absolute. The difference in liver hydroxyproline contents between groups III and IV prior to collagenase or saline perfusion raises a note of caution in interpreting the effects of collagenase in lowering hydroxyproline content. However, parallel observations of liver histology, suggest that the lowering of hydroxyproline is more likely related to lessened fibrosis than to experimental artifact. In contrast, if one were to administer collagenase to patients with viral-induced cirrhosis, the offending agent would remain and spontaneous resolution would not be a countermanding variable; in this scenario, the differences between collagenasetreated and -untreated subjects would be expected to be greater. Unknown at this time is whether fibrosis would recur once collagenase treatment was stopped in the setting of ongoing viral infection. However, even if it did, the evolution to secondary cirrhosis would take considerable time and there would then be the option to repeat collagenase portal perfusion. If treatment was extended to humans, the surgical technique would have to be refined and meticulous measures would have to be undertaken to prevent catheter leakage, as occurred in this study. Nonetheless, given the extensive and highly successful procedures being performed by transplant surgeons, the proper placement of portal catheters in humans should not represent a major challenge.

In summary, the data reported here provide evidence that portal administration of collagenase can retard cirrhosis development and increase the rate of regression of well-established liver cirrhosis in the rabbit, although some adverse effects may occur. These studies need to be expanded to a larger group of animals using more optimal techniques to reduce surgery-related mortality and using prophylactic regimens to prevent allergic reactions. The use of recombinant collagenase preparations also needs to be explored. Presently, this study is a 'proof-ofprinciple'. If the identified technical and allergic problems can be circumvented, portally administered collagenase may prove to be a new therapeutic option for patients with severe fibrosis or established cirrhosis.

\section{References}

1 Scott LF. Liver fibrosis-from bench to bedside. J Hepatol 2003;38:S38-S53.

2 Hammel P, Couvelard A, O'Toole D, et al. Regression of liver fibrosis after biliary drainage in patients with chronic pancreatitis and stenosis of the common bile duct. N Engl J Med 2001;344:418-423.

3 Wanless IR, Nakashima E, Sherman M. Regression of human cirrhosis. Morphologic features and the genesis of incomplete septal cirrhosis. Arch Pathol Lab Med 2000;124:1599-1607.

4 Iredale JP, Benyon RC, Pickering J, et al. Mechanisms of spontaneous resolution of rat liver fibrosis. Hepatic stellate cell apoptosis and reduced hepatic expression of metalloproteinase inhibitors. J Clin Invest 1998;102: 538-549.

5 Salgado S, Garcia J, Vera J, et al. Liver cirrhosis is reverted by urokinase-type plasminogen activator gene therapy. Mol Ther 2000;2:545-551.

6 Sakaida I, Hironaka K, Terai S, et al. Gadolinium chloride reverses dimethylnitrosamine (DMN)-induced rat liver fibrosis with increased matrix metalloproteinases (MMPs) of Kupffer cells. Life Sci 2003; 72:943-959.

7 Lewis DR, Burbige EJ, French SW. Reversal of cirrhosis in hemochromatosis following long-term phlebotomy. Gastroenterology 1983;83:1382 [Abstract].

8 Malekzadeh R, Mohamadnejad M, Rakhshani N, et al. Reversibility of cirrhosis in chronic hepatitis B. Clin Gastroenterol Hepatol 2004;2:344-347. 
9 Kaplan MM, DeLellis RA, Wolfe HJ. Sustained biochemical and histologic remission of primary biliary cirrhosis in response to medical treatment. Ann Intern Med 1997;126:682-688.

10 Desmet VJ, Roskams T. Cirrhosis reversal: a duel between dogma and myth. J Hepatol 2004;40:860-867.

11 Iimuro Y, Nishio T, Morimoto T, et al. Delivery of matrix metalloproteinase-1 attenuates established liver fibrosis in the rat. Gastroenterology 2003;124:445-458.

12 Siller-López F, Sandoval A, Salgado S, et al. Treatment with human metalloproteinase-8 gene delivery ameliorates experimental rat liver cirrhosis. Gastroenterology 2004;126:1122-1133.

13 Garcia-Bañuelos J, Siller-López F, Miranda A, et al. Cirrhotic rat livers with extensive fibrosis can be safely transduced with clinical-grade adenoviral vectors. Evidence of cirrhosis reversion. Gene Therapy 2002;9: 127-134.

14 Angleton EL, Van Wart HE. Preparation and reconstitution with divalent metal ions of class I and class II Clostridium histolyticum apocollagenases. Biochemistry 1988;27:7406-7412.

15 Bond MD, Van Wart HE. Purification and separation of individual collagenases of Clostridium histolyticum using red dye ligand chromatography. Biochemistry 1984;23:3077-3085.

16 Bond MD, Van Wart HE. Characterization of the individual collagenases from Clostridium histolyticum. Biochemistry 1984;23:3085-3091.

17 Kivirikko KI, Laitinen O, Prockop DJ. Modification of a specific assay for hydroxyproline in urine. Anal Biochem 1967;19:249-255.

18 Blumenkrantz N, Asboe-Hansen G. An assay for hydroxyproline and proline on one sample and a simplified method for hydroxyproline. Anal Biochem 1975;63:331-340.

19 Vargaftig BB, Lefort J, Giroux EL. Haemorrhagic and inflammatory properties of collagenase from $C$. histolyticum. Agents Actions 1976;6:627-635.

20 Poynard T, McHutchison J, Manns M, et al. Impact of pegylated interferon alfa-2b and ribavirin on liver fibrosis in patients with chronic hepatitis C. Gastroenterology 2002;122:1303-1313.

21 Dunn MA, Cheever AW, Paglia LM, et al. Reversal of advanced liver fibrosis in rabbits with Schistosomiasis japonica. Am J Trop Med Hyg 1994;50:499-505.

22 Benyon RC, Arthur MJP. Extracellular matrix degradation and the role of hepatic stellate cells. Semin Liver Dis 2001;21:373-384.
23 Perez-Tamayo R, Montfort I, Gonzalez E. Collagenolytic activity in experimental cirrhosis of the liver. Exp Mol Pathol 1987;47:300-308.

24 Kossakowska AE, Edwards DR, Lee SS, et al. Altered balance between matrix metalloproteinase and their inhibitors in experimental biliary fibrosis. Am J Pathol 1998;153:1895-1902.

25 Bruck R, Genina O, Aeed $\mathrm{H}$, et al. Halofuginone to prevent and treat thioacetamide-induced liver fibrosis in rats. Hepatology 2001;33:379-386.

26 French MF, Mookhtiar KA, Van Wart HE. Limited proteolysis of type I collagen at hyperreactive sites by class I and II Clostridium histolyticum collagenase: complementary digestion patterns. Biochemistry 1987; 26:681-687.

27 Gelbard MK, James K, Riach P, et al. Collagenase versus placebo in the treatment of Peyronie's disease: a double-blind study. J Urol 1993;149:56-58.

28 Eiler H, Hopkins FM. Successful treatment of retained placenta with umbilical cord injections of collagenase in cows. J Am Vet Med Assoc 1993;203: 436-443.

29 Rydevik B, Brown MD, Ehira T, et al. Effects of collagenase on nerve tissue. An experimental study on acute and long-term effects in rabbits. Spine 1985;10: $562-566$

30 Rydevik B, Ehira T, Linder L, et al. Microvascular response to locally injected collagenase. An experimental investigation in hamsters and rabbits. Scand J Plast Reconstr Surg Hand Surg 1989;23:17-21.

31 Hamilton RG, Mintz GR, Gelbard MK. Humoral immune responses in Peyronie's disease patients receiving clostridial collagenase therapy. J Urol 1986; 135:641-647.

32 Liu ML, Mars WM, Zarnegar R, et al. Collagenase pretreatment and the mitogenic effects of hepatocytes growth factor and transforming growth factor- $\alpha$ in adult rat liver. Hepatology 1994;19:1521-1527.

33 Recknagel RO, Glende Jr EA, Dolak JA, et al. Mechanisms of carbon tetrachloride toxicity. Pharmacol Ther 1989;43:139-154.

34 Binda D, Lasserre-Bigot D, Bonet A, et al. Time course of cytochromes $\mathrm{P} 450$ decline during rat hepatocyte isolation and culture: effect of L-NAME. Toxicol In Vitro 2003;17:59-67.

35 Eeckhoutte C, Albo AG, Carletti M, et al. Timedependent variations of drug-metabolising enzyme activities (DMEs) in primary cultures of rabbit hepatocytes. Toxicol In Vitro 2002;16:375-382. 